\title{
Why the Coriolis force turns a wind farm wake clockwise in the Northern Hemisphere
}

\author{
Maarten Paul van der Laan and Niels Nørmark Sørensen \\ Technical University of Denmark, DTU Wind Energy, Ris $\varnothing$ Campus, Frederiksborgvej 399, \\ 4000 Roskilde, Denmark \\ Correspondence to: Maarten Paul van der Laan (plaa@dtu.dk)
}

Received: 9 December 2016 - Discussion started: 14 December 2016

Revised: 21 March 2017 - Accepted: 27 April 2017 - Published: 29 May 2017

\begin{abstract}
The interaction between the Coriolis force and a wind farm wake is investigated by Reynoldsaveraged Navier-Stokes simulations, using two different wind farm representations: a high roughness and $5 \times 5$ actuator disks. Surprisingly, the calculated wind farm wake deflection is the opposite in the two simulations. A momentum balance in the cross flow direction shows that the interaction between the Coriolis force and the $5 \times 5$ actuator disks is complex due to turbulent mixing of veered momentum from above into the wind farm, which is not observed for the interaction between the Coriolis force and a roughness change. When the wind farm simulations are performed with a horizontally constant Coriolis force in order to isolate the effect of the wind veer, the wind farm wake deflection of the $5 \times 5$ actuator disks simulation remains unchanged. This proves that the present wind veer deflects the wind farm wake and not the local changes in the Coriolis force in the wake deficit region. An additional simulation of a single actuator disk, operating in a shallow atmospheric boundary layer, confirms that the Coriolis force indirectly turns a wind turbine wake clockwise, as observed from above, due to the presence of a strong wind veer.
\end{abstract}

\section{Introduction}

In recent years, wind farms have grown in size and are more frequently placed in wind farm clusters. This means that large-scale effects are becoming more important for wind turbine wake interaction in wind farms, and especially for the interaction between wind farms. One large-scale effect that is often neglected by wind farm modelers is the effect of the Coriolis force on wind turbine or farm wakes. In previous work (van der Laan et al., 2015a), we have shown that the Coriolis force should not be neglected in Reynoldsaveraged Navier-Stokes (RANS) simulations of a wind farm cluster consisting of two wind farms in a neutrally stratified atmospheric boundary layer (ABL). The deflection of the upstream wind farm wake resulted in a lower power production of the downstream wind farm because the Coriolis force aligned the upstream wind farm wake towards the curved wind turbine rows of the downstream wind farm. Note that a constant latitude was used, which means that the global turning of the Coriolis force was not modeled. In other words, only the interaction between the Coriolis force and local disturbances in the velocity field were investigated. In the present work, we will also use a constant latitude.

The literature does not agree on the turning direction of wind farm wakes caused by the Coriolis force. Volker et al. (2015) showed that mesoscale models with different wind farm parameterizations can show wind farm wake deflections in opposite directions for the same test case. Magnusson and Smedman (1994) have observed that a strong wind veer, caused by the Coriolis force and more pronounced in stable atmospheric conditions, can lead to a skewed wind turbine wake. Several authors have used large eddy simulation (LES) to investigate this effect on wind turbines and wind farms. LES of Lu and Porté-Agel (2011) confirmed that a strong wind veer can lead to a skewed wind turbine wake, which is observed as a clockwise rotation at hub height, in the Northern Hemisphere. Here, we define the wake deflection as clockwise and anticlockwise, as observed from above, and for simplicity we only discuss wind farms located in 
the Northern Hemisphere. More recently, Churchfield et al. (2016) and Abkar and Porté-Agel (2016) have shown similar results using LES of wakes in a stable ABL. By contrast, Dörenkämper et al. (2015) reported a small anticlockwise deflection in a LES of a wind farm wake in a stable ABL. Dörenkämper et al. (2015) generated the ABL with a precursor simulation using a different roughness length compared to the one applied in the wind farm simulation in order to model coast effects on an offshore wind farm. This means that the inlet ABL profile develops downstream and could have impacted the wind farm wake deflection. Allaerts and Meyers (2017) also observed a small anticlockwise wind farm deflection $\left(2^{\circ}\right)$ in LES of a wind farm in a neutral ABL. However, the observed turning is so small that it becomes challenging to extract it from a LES data set. Even though Allaerts and Meyers (2017) have used a precursor simulation with the same the roughness length as used in the wind farm simulation, the inserted $\mathrm{ABL}$ at the inlet can still develop downstream, which can lead to small wind direction changes in the wind farm. This is because the generated ABL from the precursor simulation in LES is only in a pseudo steady state. In our RANS simulations (van der Laan et al., 2015a), the neutral ABL is in steady state and it is in balance with the entire domain. Another problem of simulating the interaction between the Coriolis force and a wind farm wake in LES is that one might need to simulate a very long time in order to obtain a statistically independent average of a small quantify such as the wind direction deflection in neutral atmospheric conditions.

Mitraszewski (2012) argued that a wind farm can be seen as a roughness change, and therefore the Coriolis force should turn the wind farm wake anticlockwise, following Orr et al. (2005). In contradiction to Mitraszewski (2012), it was shown in previous work (van der Laan et al., 2015a) that the Coriolis force turns a wind farm wake clockwise (in neutral atmospheric conditions), and this is explained as a result of a stream-wise decreasing Coriolis force in the wake recovery region. In summary, the interaction between the Coriolis force and the wind farm wake is explained in the literature in two different ways:

1. The Coriolis forces change in the wake region and induce a clockwise (van der Laan et al., 2015a) or anticlockwise (Mitraszewski, 2012; Dörenkämper et al., 2015; Allaerts and Meyers, 2017) wake defection.

2. The present wind veer deflects the wind farm wake clockwise (Magnusson and Smedman, 1994; Lu and Porté-Agel, 2011; Churchfield et al., 2016; Abkar and Porté-Agel, 2016).

Our goal is to clarify why the Coriolis force turns a wind farm wake clockwise in the Northern Hemisphere. First, we test the hypothesis of Mitraszewski (2012) by performing two wind farm simulations with RANS in a neutrally stratified $\mathrm{ABL}$, where the wind farm is represented in two dif- ferent ways: using actuator disks (ADs) (Mikkelsen, 2003) and using a high roughness in the wind farm area. Secondly, the two wind farm simulations are repeated using a Coriolis force that is set as constant in horizontal directions in order to remove the influence of local changes in the Coriolis force in the wake region and to isolate the effect of the present wind veer on the wind farm wake deflection. In addition, we investigate the wake deflection of a single AD placed in a shallow ABL, where the wind veer over the rotor area is large, to see if our RANS model agrees with LES results from Lu and Porté-Agel (2011), Churchfield et al. (2016), Abkar and Porté-Agel (2016), and field measurements of Magnusson and Smedman (1994).

Note that this work is an extension of van der Laan and Sørensen (2016), presented at the TORQUE 2016 conference.

\section{Methodology}

In order to understand the interaction between the Coriolis force and a wind farm wake, two RANS simulations of a simple rectangular wind farm of $5 \times 5$ NREL $5 \mathrm{MW}$ wind turbines (Jonkman et al., 2009) are carried out, where the wind farm is represented in two different ways:

1. 25 wind turbines represented by ADs (Mikkelsen, 2003) with variable forces (van der Laan et al., 2015b), without wake rotation

2. a high roughness of $1 \mathrm{~m}$ in the wind farm area.

The NREL $5 \mathrm{MW}$ wind turbine has a hub height $\left(z_{\mathrm{H}}\right)$ and a rotor diameter $(D)$ of 90 and $126 \mathrm{~m}$, respectively. The wind turbines spacing is set to $8 D$ in both horizontal directions. In addition, a third RANS simulation of a single NREL $5 \mathrm{MW}$ wind turbine in a shallow ABL is carried out, where the wind turbine is represented by an $\mathrm{AD}$.

In the simulation where the wind farm is represented by a high roughness, a roughness length of $1 \mathrm{~m}$ is chosen. Frandsen et al. (2009) and Volker (2014) have used values between 0.5 and $0.7 \mathrm{~m}$, using the wind farm roughness length relation of Frandsen (2007). Calaf et al. (2010) performed LES of a fully developed boundary layer over an infinitely large wind farm and showed that the wind farm roughness length can be as high as $8.9 \mathrm{~m}$. Hence, using $1 \mathrm{~m}$ as a wind farm roughness length in the present work seems to be in the correct range. Choosing a different roughness length will change the results but not the general trends in wind farm wake deflection.

The numerical setup of the RANS simulations with ADs including the Coriolis force is fully described in previous work (van der Laan et al., 2015a), and we will briefly summarize it here. The simulations are carried out with the in-house flow solver EllipSys3D developed by Sørensen (1994) and Michelsen (1992). The turbulence is modeled with a modified $k-\varepsilon$ model that limits the boundary layer height through a 

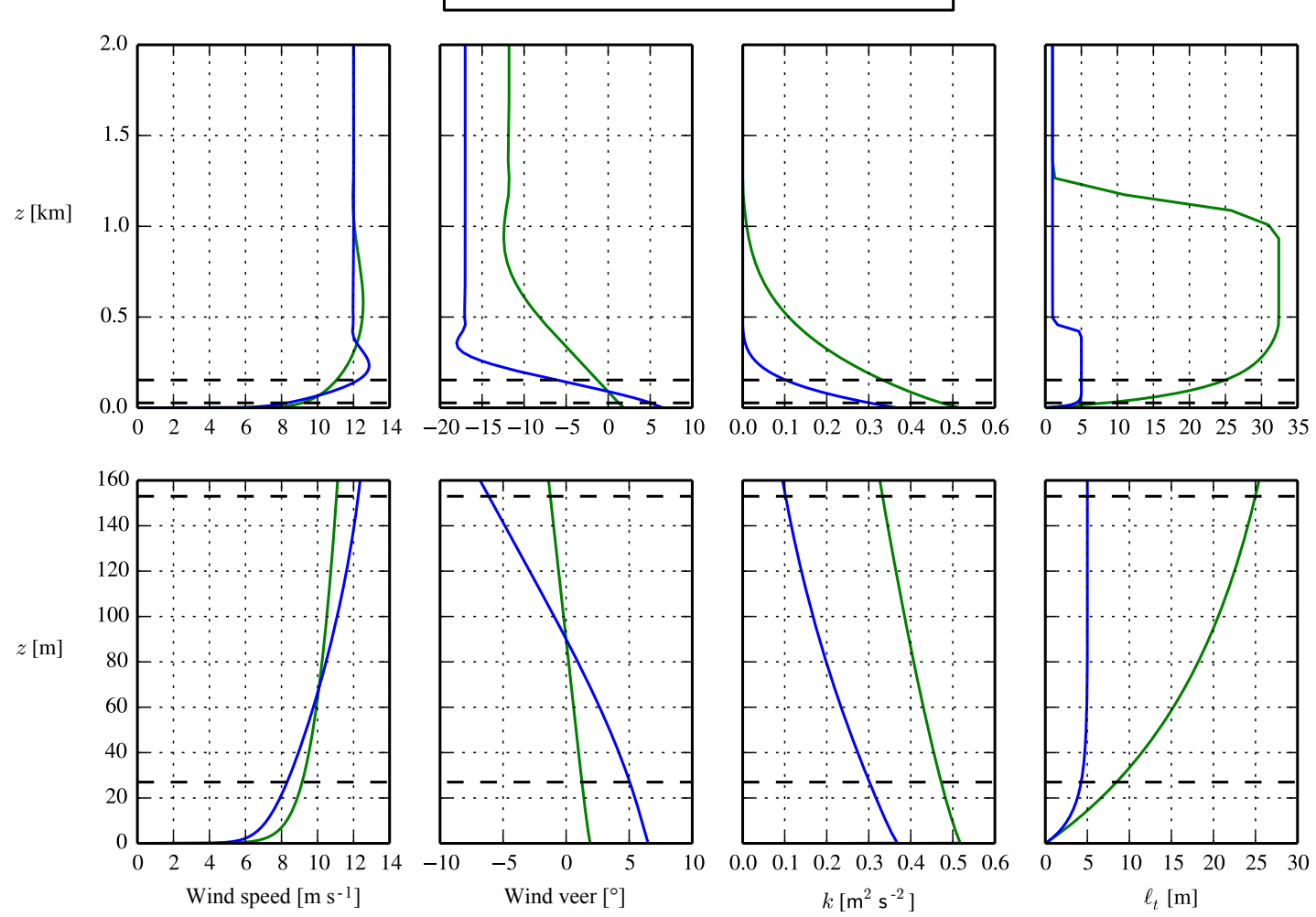

Figure 1. Rotated inlet profiles calculated by the precursor. Bottom plots are a zoomed view of the top plots. Rotor area is shown as black dashed lines.

global length scale limiter as introduced by Apsley and Castro (1997), and it includes a local length scale limiter that is necessary to resolve the near wind turbine wake properly (van der Laan et al., 2015c). The inflow profiles of the wind farm simulations are determined from a neutrally stratified precursor simulation, where the Coriolis force is balanced by a defined pressure gradient; both terms are implemented as a momentum source term $S_{v}$ :

$S_{v, x}=\rho f_{\mathrm{c}}\left(V-V_{G}\right), \quad S_{v, y}=-\rho f_{\mathrm{c}}\left(U-U_{G}\right)$,

with $\rho$ as the air density, $f_{\mathrm{c}}$ as the Coriolis parameter set to $10^{-4} \mathrm{~s}^{-1}, U$ and $V$ are the stream-wise and lateral velocity components, and the subscript $G$ denotes the geostrophic wind, which is set to $12 \mathrm{~m} \mathrm{~s}^{-1}$. A uniform roughness length of $10^{-4} \mathrm{~m}$ is chosen. In the wind farm simulations, the maximum turbulent length scale $\ell_{t, \max }$ used in the global length scale limiter is based on Blackadar (1962):

$\ell_{\mathrm{t}, \max }=0.00027 \frac{G}{f_{\mathrm{c}}}$,

which gives an $\ell_{t, \max }$ of $32.4 \mathrm{~m}$. In the single-AD simulation, $\ell_{t, \max }$ is set to $5 \mathrm{~m}$ to enforce a shallow ABL with a strong wind veer over the rotor area. The shallow ABL could be seen as a pseudo stable ABL, where the direct modeling of buoyancy in the momentum and turbulence equations, as performed in the RANS setup of Sogachev et al. (2012) and Koblitz et al. (2015), is neglected. The precursor simulation of the wind farm test cases $\left(\ell_{\mathrm{t}, \mathrm{max}}=32.4 \mathrm{~m}\right)$ calculates a velocity of $10.4 \mathrm{~m} \mathrm{~s}^{-1}$ and a turbulence intensity of $5 \%$ at hub height, which represents neutral off-shore conditions. The velocity and a turbulence intensity at hub height for the precursor simulation of the single $\mathrm{AD}$ in a shallow $\operatorname{ABL}\left(\ell_{\mathrm{t}, \max }=5 \mathrm{~m}\right)$ is equal to $10.8 \mathrm{~m} \mathrm{~s}^{-1}$ and $3.2 \%$, respectively. The calculated profiles include wind veer, and are rotated to enforce a chosen row aligned to a wind direction of $270^{\circ}$ at hub height in the wind farm simulations. The same wind direction is used in the single-AD case. The rotated inflow profiles from both precursor simulations are shown in Fig. 1. A wind veer of about 2.5 and $11^{\circ}$ are present in the rotor area, as shown in one of the bottom plots of Fig. 1.

The same numerical grid is used in both wind farm simulations. The domain definition including wind farm layout and boundary conditions (BCs) is shown in Fig. 2. The grid is Cartesian and represents a box-shaped domain with dimensions $2000 D \times 1000 D \times 20 D$ in stream-wise, lateral and vertical directions, respectively. The grid consist of 22 million cells, where the horizontal spacing in and around the wind farm (inside the blue rectangle of Fig. 2) is $D / 8$. Inside the red rectangle of Fig. 2, the grid cells are stretched in the stream-wise direction towards a spacing of $1 D$, up 

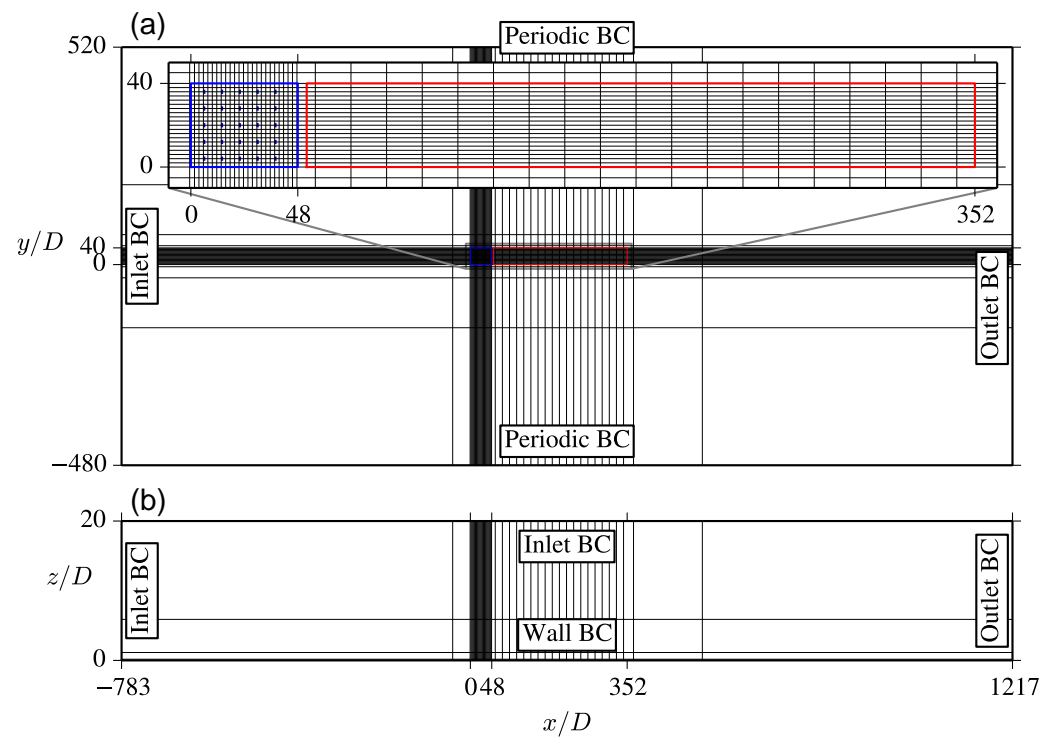

Figure 2. Grid and boundary conditions of wind farm simulations. Every 16th grid line is plotted. (a) Top view of the grid, including a zoomed view. (b) Side view of the grid. Blue filled boxes represent ADs. Spacing inside the blue and red rectangles are set to $D / 8$ and $D$, respectively.
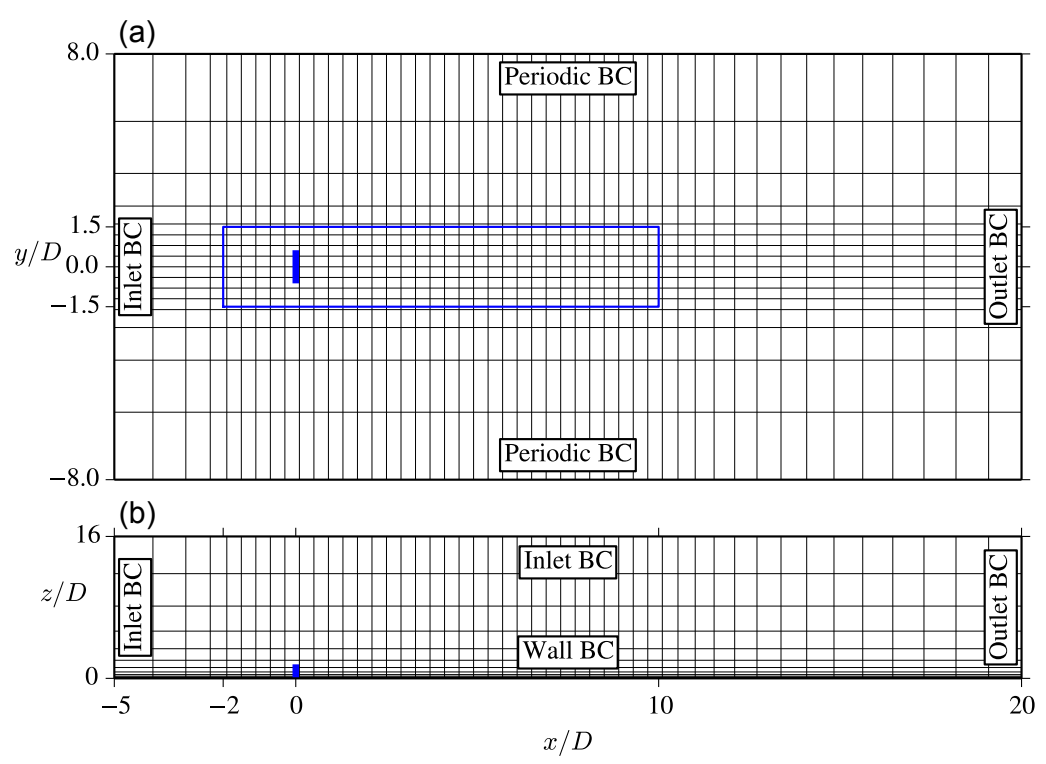

Figure 3. Grid and boundary conditions of the single-AD simulation. Every fourth grid line is plotted. (a) Top view of the grid. (b) Side view of the grid. Blue filled box represents the AD. The horizontal spacing inside the blue rectangle is set to $D / 8$.

to $352 D \approx 44 \mathrm{~km}$ downstream. The vertical resolution starts with a cell height of $0.5 \mathrm{~m}$, and it grows with height using an expansion ratio of about 1.1. The profiles from the precursor simulation are inserted at the inlet BC, as shown in Fig. 2. In addition, the top boundary of the domain is also an inlet $\mathrm{BC}$. The lateral boundaries are periodic to account for wind veer. At the outlet BC, a fully developed flow is assumed. A rough wall BC (Sørensen et al., 2007) is placed at the bottom of the domain.
The numerical grid and boundary conditions of the single $\mathrm{AD}$ are shown Fig. 3. The domain has dimensions $25 D \times 16 D \times 16 D$ in stream-wise, lateral and vertical directions, respectively. The horizontal grid spacing inside the blue rectangle with dimensions $12 D \times 3 D$ is set to $D / 8$. A total number of $7.9 \times 10^{5}$ cells are used in the grid. The boundary conditions of the wind farm simulations are also applied to the single-AD simulation. 
(a)

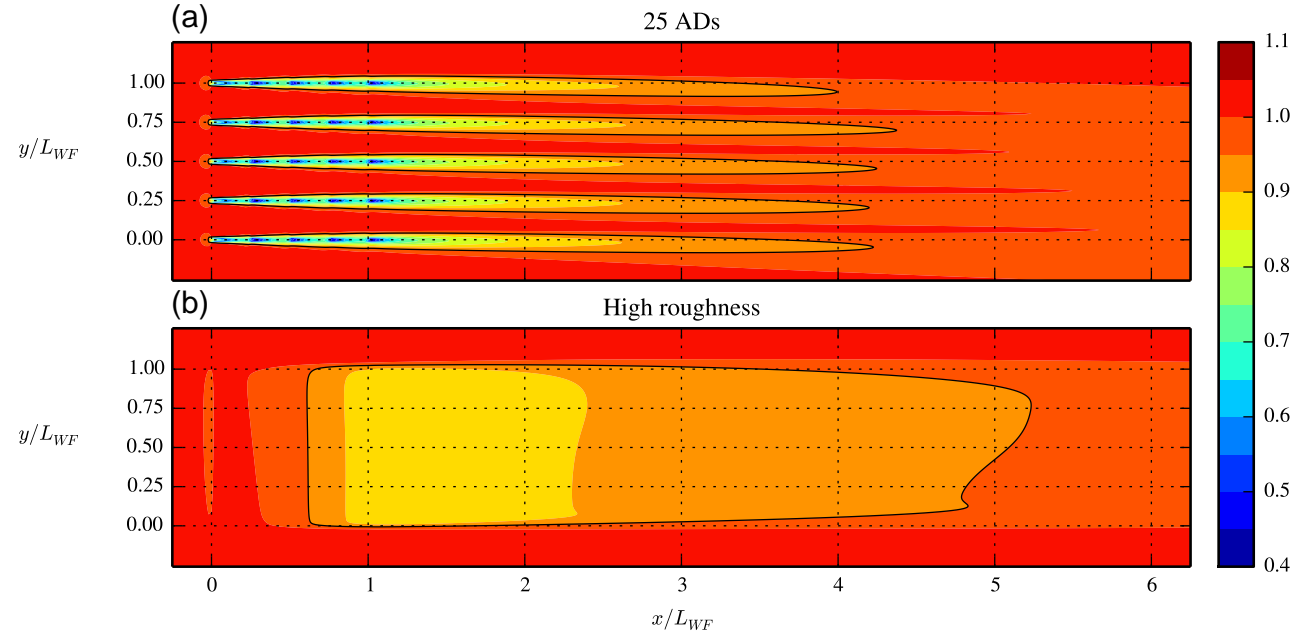

Figure 4. Stream-wise velocity at hub height, normalized by the free stream. (a) Wind farm modeled with 25 ADs. (b) Wind farm modeled as a high roughness. Contour line represents $95 \%$ recovered velocity.

\section{Results and discussion}

\subsection{Wind farm simulations in a neutral $\mathrm{ABL}$}

Figure 4 shows two contour plots of the stream-wise velocity, taken at hub height, for the two wind farm representations: $25 \mathrm{ADs}$ and a high roughness. The $x$ and $y$ coordinates are normalized by the length or width of the wind farm $L_{\mathrm{WF}}=32 \mathrm{D}$. The simulation with $25 \mathrm{ADs}$ shows five distinct merged wakes, while the simulation of the roughness change shows one wind farm wake structure. A contour line that represents $95 \%$ recovered velocity is shown in each plot of Fig. 4. The contour line reveals that the near wake of the wind farm represented by ADs is deflected clockwise, while the opposite is observed for the wind farm represented by a high roughness.

In Fig. 5, the turbulence intensity at hub height is plotted for both wind farm representations. The turbulence intensity is larger and more concentrated for the wind farm represented by the $25 \mathrm{ADs}$ compared to the wind farm represented by the high roughness. In addition, the increase in turbulence intensity at hub height is delayed in the roughness change simulation because the internal boundary layer (IBL) starts at $z=z_{0}$ (instead of $z=z_{\mathrm{H}}$ ).

The wind farm wake deflection is also visible in Fig. 6, where contours of the stream-wise velocity, subtracted and normalized by the free stream $\left(U-U_{\text {inflow }}\right) / U_{\text {inflow }}$, are plotted at five cross planes located at $x / L_{\mathrm{WF}}=0,0.1,1,2,6$ for both simulations. Figure 6 shows that the far wake $\left(x / L_{\mathrm{WF}}=\right.$ 6 ) of the wind farm represented by a high roughness turns back towards the free stream, since an opposite roughness change occurs after the wind farm $\left(1 \mathrm{~m} \rightarrow 10^{-4} \mathrm{~m}\right)$. The clockwise wake deflection of the wind farm represented by the $25 \mathrm{ADs}$ is still visible at $x / L_{\mathrm{WF}}=6$.

\subsubsection{Momentum balance}

In this section, the observed wind farm wake deflection from Figs. 4 and 6 is explained using a momentum balance.

The momentum equation can be written as

$$
\frac{D U_{i}}{D t}=S_{\mathrm{AD}, i}+\frac{1}{\rho}\left(S_{v, i}-\frac{\partial \widetilde{P}}{\partial x_{i}}\right)-\frac{\partial \overline{u_{i}^{\prime} u_{j}^{\prime}}}{\partial x_{j}} .
$$

Here, we neglect the molecular viscosity since it is much smaller than the eddy viscosity. $S_{\mathrm{AD}, i}$ represents the $\mathrm{AD}$ forces in the wind farm simulation including ADs, and $\widetilde{P}$ represents the fluctuation around the static pressure that is solved by the SIMPLE algorithm from (Patankar and Spalding, 1972). The pressure gradients are obtained as $\partial P / \partial x=$ $\partial \widetilde{P} / \partial x+\rho f_{\mathrm{c}} V_{G}$ and $\partial P / \partial y=\partial \widetilde{P} / \partial y-\rho f_{\mathrm{c}} U_{G}$. We are interested in the momentum balance in the cross direction $(y)$, which can be written as

$$
\begin{aligned}
& \int_{A} \frac{D V}{D t} \mathrm{~d} A \\
& \text { Imbalance of V-momentum } \\
& =-f_{\mathrm{c}} \int_{A} U \mathrm{~d} A-\frac{1}{\rho} \int_{A} \frac{\partial P}{\partial y} \mathrm{~d} A \quad-\int_{A} \frac{\partial \overline{v^{\prime} w^{\prime}}}{\partial z} \mathrm{~d} A \\
& \text { Coriolis Pressure gradient Turbulence }
\end{aligned}
$$

The integrals are taken over square horizontal slices with an area of $A=40 \times 40 D^{2}$ at several heights, in the wind farm $\left(x=y=\left\{-4 D, L_{\mathrm{WF}}+4 D\right\}\right)$ and in the near wind farm wake $\left(x=\left\{L_{\mathrm{WF}}, 2 L_{\mathrm{WF}}+8 D\right\}, y=\left\{-4 D, L_{\mathrm{WF}}+4 D\right\}\right)$. The integrals $\int_{A} \frac{\partial \overline{u^{\prime} v^{\prime}}}{\partial x} \mathrm{~d} A$ and $\int_{A} \frac{\partial \overline{v^{\prime} v^{\prime}}}{\partial y} \mathrm{~d} A$ are neglected because they are 2-3 orders of magnitude smaller than the other integrals from Eq. (4). In addition, $S_{\mathrm{AD}, y}=0$ since only thrust forces are considered, and the ADs are fixed normal to the flow direction. Each term of Eq. (4) is normalized by $1 /\left(A f_{\mathrm{c}} G\right)$ and plotted in Fig. (7). 


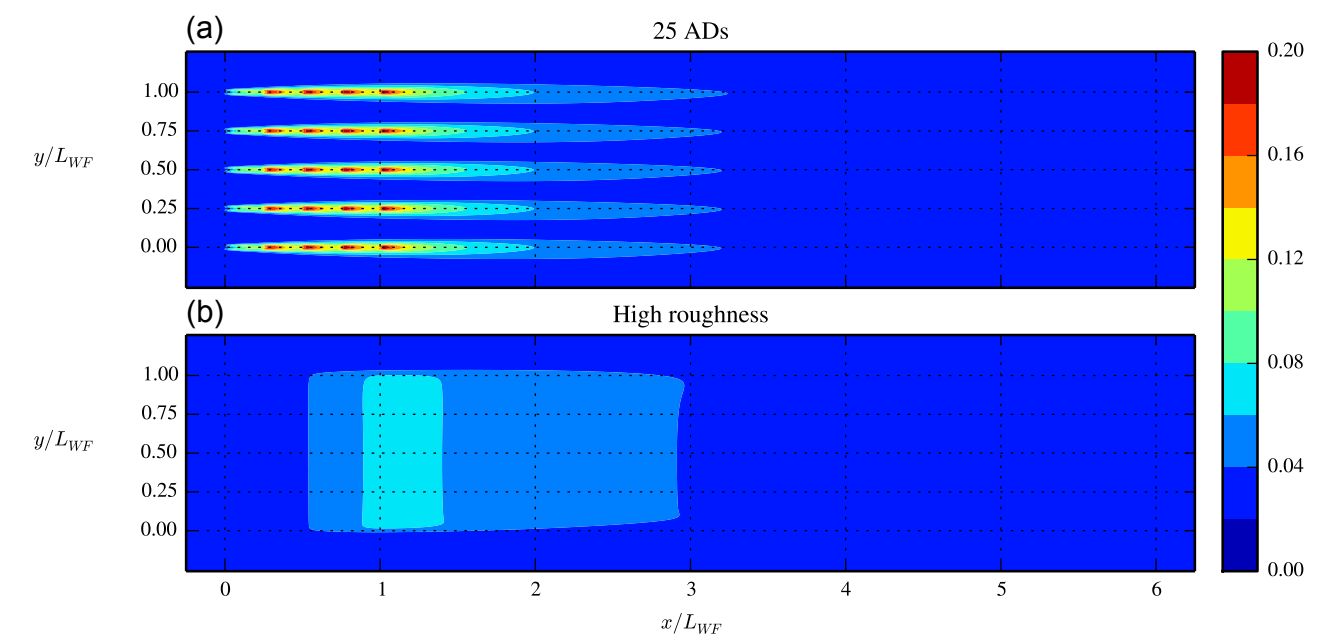

Figure 5. Turbulence intensity at hub height. (a) Wind farm modeled with 25 ADs. (b) Wind farm modeled as a high roughness.

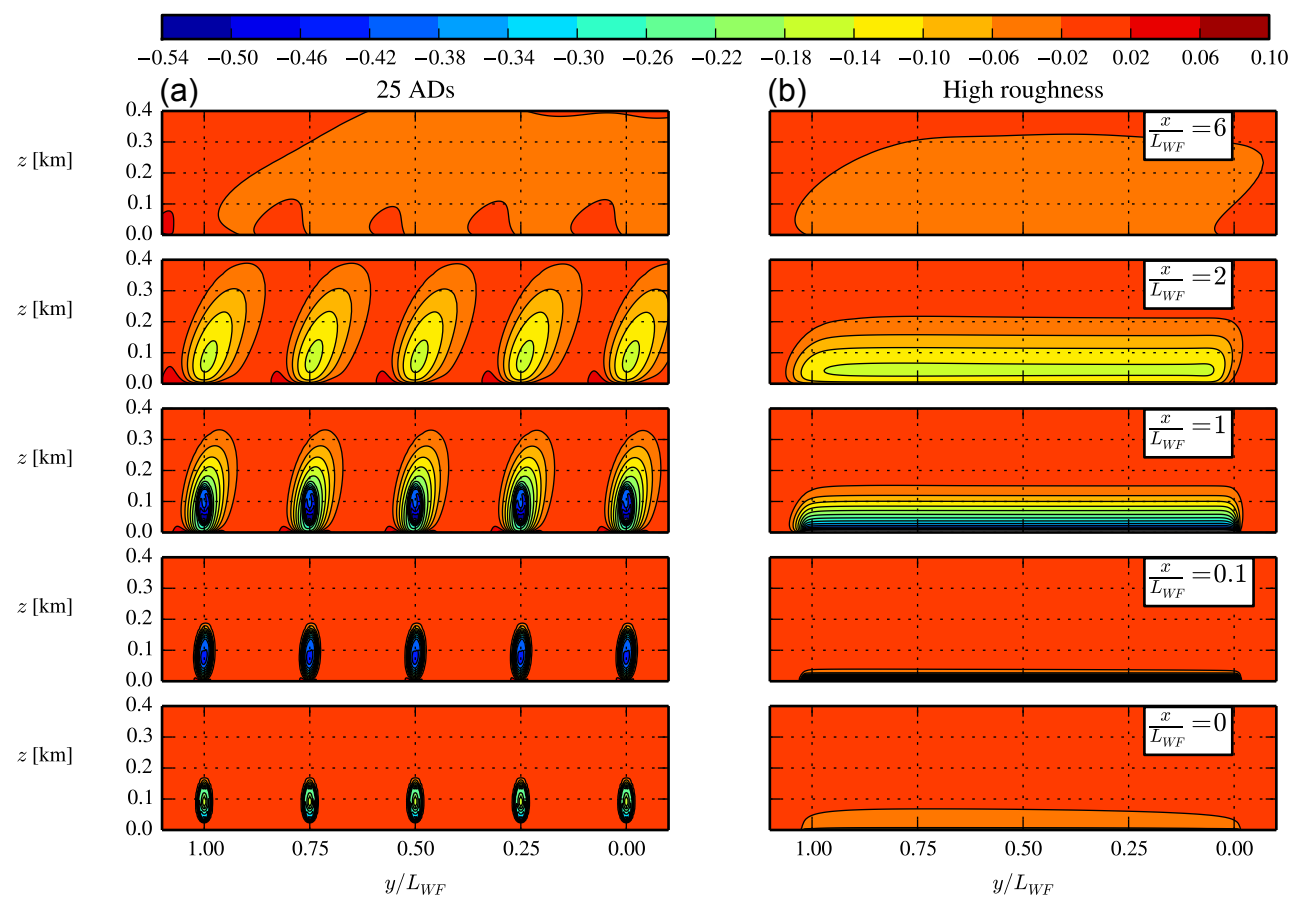

Figure 6. Stream-wise velocity subtracted and normalized by the free stream $\left(U-U_{\text {inflow }}\right) / U_{\text {inflow }}$ at several downstream cross planes. (a) Wind farm is represented by 25 ADs. (b) Wind farm is represented by a high roughness. Wind farm ends at $x / L_{\mathrm{WF}}=1$.

The top and bottom figures show results from the simulation where the wind farm is represented by $25 \mathrm{ADs}$ and a high roughness, respectively. In addition, the results from the left figures are taken from inside the wind farm, while the right figures are made on the basis of the near wind farm wake. The results from the wind farm simulation (solid lines) are compared with the results taken from an empty domain (colored dashed lines). When the wind farm is not present (colored dashed lines from Fig. 7), the turbulence is in balance with the Coriolis force and pressure gradient, as expected. At the wind farm area (Fig. 7a, c), there is more turbulence developing when the wind farm is represented by ADs (Fig. 7a) compared to a wind farm represented by a roughness change (Fig. 7c). In the near wind farm wake (Fig. 7b, d), this difference in turbulence between the two wind farm simulations is even more pronounced.

When the wind farm is represented by a high roughness (Fig. 7c, d), an IBL develops from the abrupt roughness change at $x / L_{\mathrm{WF}}=0$. The largest changes in turbulence mainly occur near the wall due to the IBL, where the 


\begin{tabular}{|c|c|c|c|c|c|c|}
\hline $\begin{array}{l}\text { Empty domain: } \\
\text { Wind farm: }\end{array}$ & - & $\begin{array}{l}\text { Imbalance of } \\
\mathrm{V} \text { momentum }\end{array}$ & - & $\begin{array}{l}\text { Coriolis and } \\
\text { pressure gradient }\end{array}$ & - & Turbulence \\
\hline
\end{tabular}
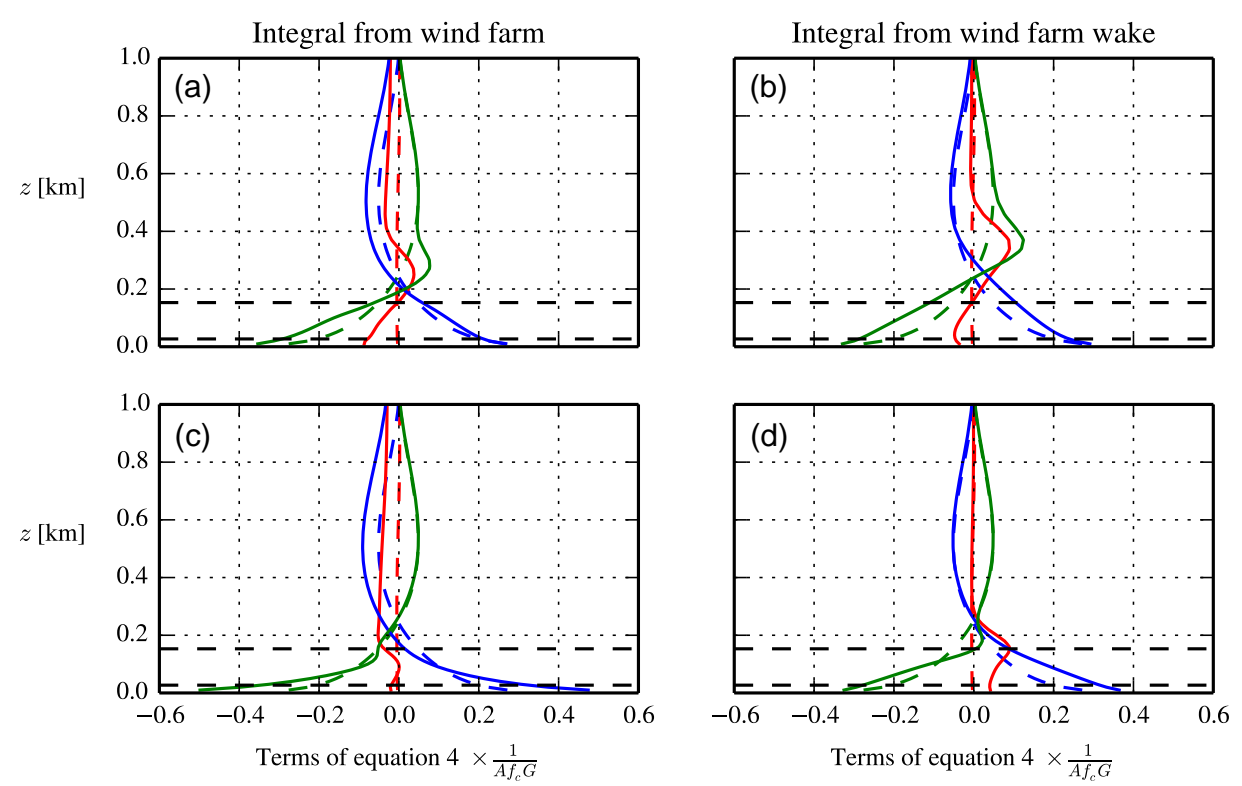

Figure 7. $V$-Momentum balance. (a, b) Wind farm represented by 25 ADs, where rotor area is shown as black dashed lines. (c, d) Wind farm represented by a high roughness. (a, c) Integral taken from wind farm area. (b, d) Integral taken from near wind farm wake. Dashed lines represent the results from an empty domain.

largest imbalance of $V$ momentum in the near wind farm wake (Fig. 7d) is also observed. In addition, the near wake (Fig. 7d) shows that the combined change in Coriolis force and pressure gradient is larger than the change in the turbulence. Hence, the local changes in Coriolis force and pressure gradient deflect the wind farm wake anticlockwise, which is already visible in the wind farm area, as shown by Fig. $6 \mathrm{~b}$.

When the wind farm is represented by $25 \mathrm{ADs}$ (Fig. 7a, b), the turbulence and $V$ momentum change both near the wall and above the wind turbines. In both the wind farm and the near wind farm wake, the change in turbulence is larger than the combined change in Coriolis force and pressure gradient, especially above the wind farm, where also the imbalance of $V$ momentum in the near wind farm wake is the largest. This indicates that the turbulence mixes flow from above the wind farm that has a relative wind direction towards the right, down into the wake region, which causes the wind farm wake to turn clockwise. In other words, Fig. 7 suggests that the Coriolis force indirectly causes the wind farm wake to deflect clockwise because of the present wind veer and not because of the local changes in the Coriolis force as suggested in previous work (van der Laan et al., 2015a).

Figure 7 shows that the flow in a simulation with 25 ADs including Coriolis is complex and very different from a simulation modeling a roughness change with Coriolis force. This means that the interaction between the Coriolis force and a wind farm wake cannot be simplified to the inter- action between the Coriolis force and a roughness change when the wake deflection is investigated, as suggested by Mitraszewski (2012).

\subsubsection{Constant vs. variable Coriolis forces}

One could set the Coriolis force source terms to be constant in the horizontal directions (also at the wind farm) to isolate the effect of the wind veer on the wind farm wake deflection:

$$
\begin{aligned}
& S_{v, x}=\rho f_{\mathrm{c}}\left(V_{\text {precursor }}-V_{G}\right), \\
& S_{v, y}=-\rho f_{\mathrm{c}}\left(U_{\text {precursor }}-U_{G}\right),
\end{aligned}
$$

where $U_{\text {precursor }}$ and $V_{\text {precursor }}$ are taken from the precursor simulation of the ABL profiles that are also used at the inlet boundary. (Without wind farm, the ABL profiles are maintained throughout the domain.) The stream-wise velocity contours at hub height are plotted in Figs. 8 and 9 for a wind farm represented by 25 ADs and a high roughness, respectively. In each figure, results with a variable (as already shown in Fig. 4) and a constant Coriolis force (Eq. 5) are plotted. Figure 8 shows that there is hardly any visible difference in the wind farm wake deflection between a constant and a variable Coriolis force, which means that only the wind veer can be causing the clockwise wind farm wake deflection in a wind farm represented by $25 \mathrm{ADs}$. When the wind farm is modeled as a high roughness, as depicted in Fig. 9, the difference between a constant and a variable Coriolis force is 


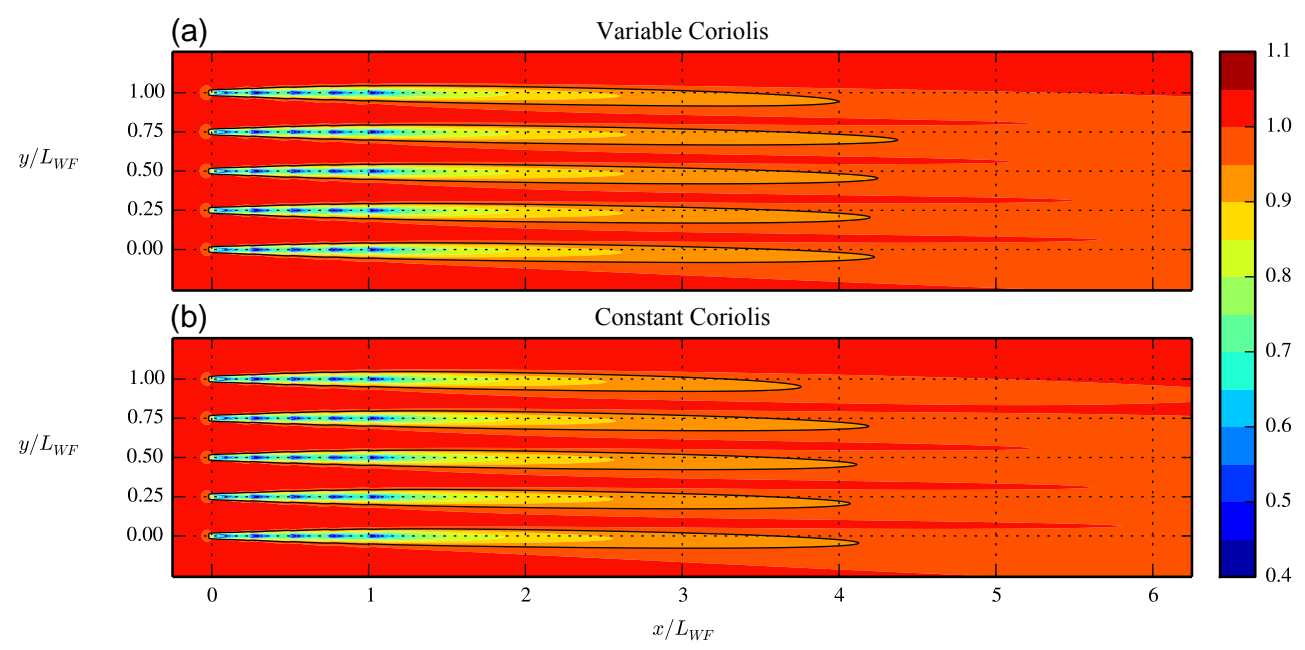

Figure 8. Stream-wise velocity at hub height, normalized by the free stream. Wind farm represented by 25 ADs. Contour line represents $95 \%$ recovered velocity.

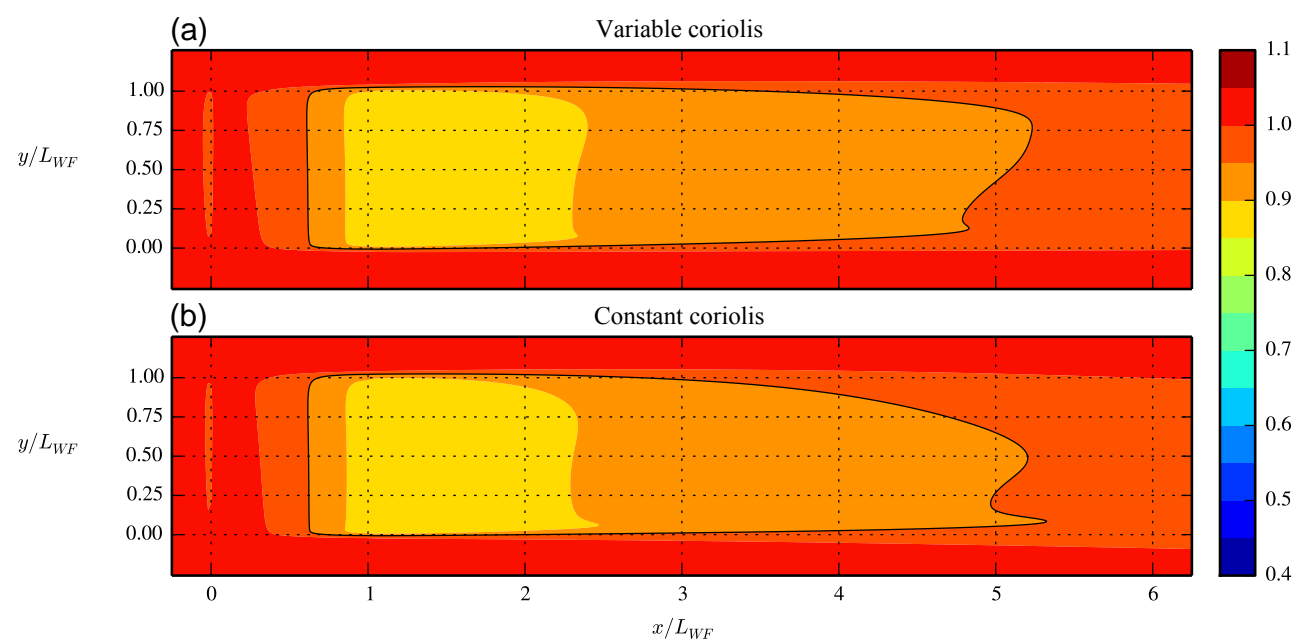

Figure 9. Stream-wise velocity at hub height, normalized by the free stream. Wind farm represented by a high roughness. Contour line represents $95 \%$ recovered velocity.

clearly visible. For a constant Coriolis force, the wake of the wind farm represented by the high roughness deflects more clockwise compared to the variable Coriolis force because a varying Coriolis force turns the wind farm wake anticlockwise, while the wind veer does the opposite. This shows that the locally changing Coriolis force is important in the simulation of the high-roughness wind farm. Overall, Fig. 8 is proof that the wind veer turns the wake of wind farm represented by 25 ADs clockwise, as previously concluded from the results of the momentum balance discussed in Sect. 3.1.1.

\subsection{Single-wake simulation in a shallow $A B L$}

Contours of stream-wise velocity, subtracted and normalized by the free stream, of a single AD operating in a shallow $\mathrm{ABL}$ are shown in Fig. 10 for four downstream cross planes located at $x / D=0,2,4,10$. Figure 10 shows that the strong wind veer stretches the wind turbine wake, which is observed as a clockwise rotation at hub height, as shown in Fig. 11. A similar result has been observed in field measurements (Magnusson and Smedman, 1994) and LES of a single wind turbine and wind farms in a stable ABL (Lu and Porté-Agel, 2011; Churchfield et al., 2016; Abkar and Porté-Agel, 2016). The single-AD test case confirms that the Coriolis force indirectly deflects a wind turbine or farm wake clockwise because of the wind veer. Note that in neutral conditions, the wake deflection of single $\mathrm{AD}$ due to Coriolis is negligible because the wind veer is not strong enough, as shown in previous work (van der Laan et al., 2015a). 


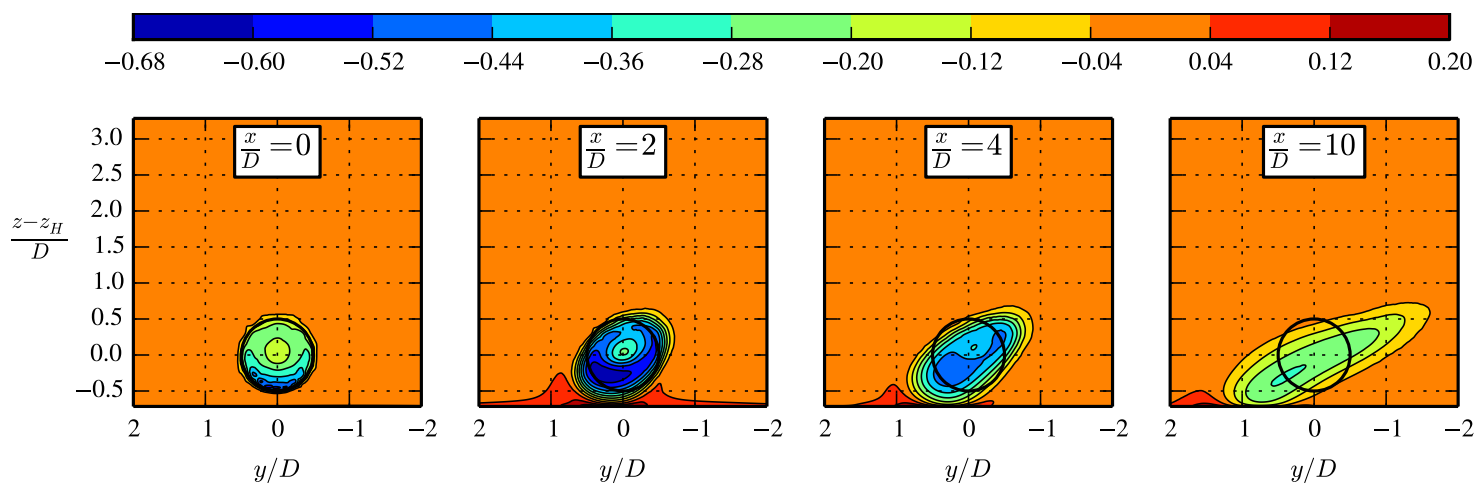

Figure 10. Stream-wise velocity subtracted and normalized by the free stream $\left(U-U_{\text {inflow }}\right) / U_{\text {inflow }}$ at several downstream cross planes for a single wake in a shallow ABL. AD is shown as a black circle.

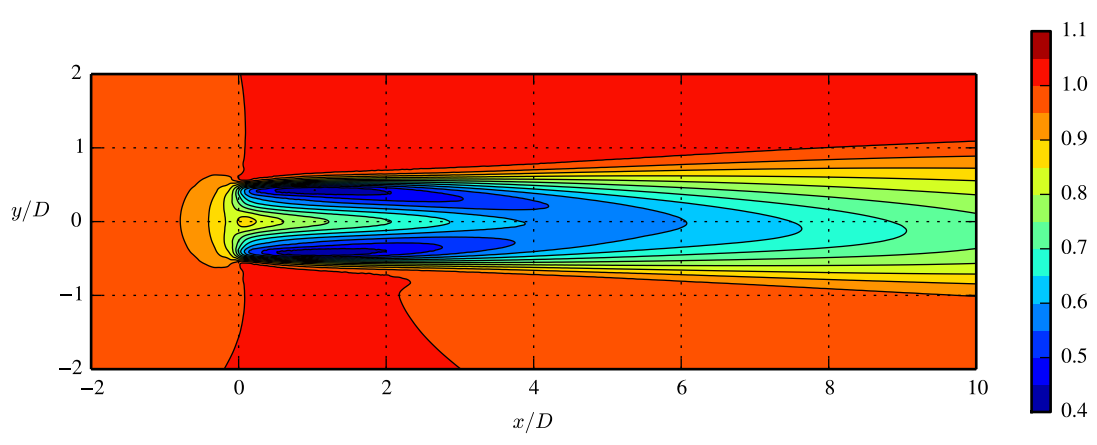

Figure 11. Stream-wise velocity at hub height, normalized by the free stream, for a single wake in a shallow ABL.

\section{Conclusions}

Two RANS simulations of a wind farm including the effect of the Coriolis force are carried out that differ in wind farm representation. When the wind farm is modeled as a roughness change, the wind farm wake turns anticlockwise due to an imbalance in the Coriolis force. When the wind farm is represented by 25 actuator disks, the wind farm wake is deflected clockwise. An investigation of the momentum balance in the cross flow direction suggests that in the simulation with 25 actuator disks, the turbulence mixes momentum from above that has a relative wind direction towards the right, down into the wake region. When the Coriolis force is set as constant in the horizontal dimensions to isolate the effect of wind veer, the wind farm wake deflection of the 25 actuator disks is unaffected. This proves that the Coriolis force indirectly causes the wind farm wake to deflect clockwise because of the present wind veer and not because of the local changes in the Coriolis force; this is also confirmed by the simulation of a single actuator disk operating in a shallow atmospheric boundary layer. Hence, the interaction between the Coriolis force and a wind farm wake is a complex process that cannot be simplified to the interaction between the Coriolis force and a roughness change when the deflection of the wind farm wake is investigated.
Data availability. The data presented can be made available by contacting the corresponding author.

Competing interests. The authors declare that they have no conflict of interest.

Acknowledgements. This work is supported by the Center for Computational Wind Turbine Aerodynamics and Atmospheric Turbulence funded by the Danish Council for Strategic Research, grant number 09-067216. Computational resources were provided by DCSC and the DTU central computing facility.

Edited by: C. L. Bottasso

Reviewed by: two anonymous referees

\section{References}

Abkar, M. and Porté-Agel, F.: Influence of the Coriolis force on the structure and evolution of wind turbine wakes, Phys. Rev. Fluids, 1, 1-14, doi:10.1103/PhysRevFluids.1.063701, 2016.

Allaerts, D. and Meyers, J.: Boundary-layer development and gravity waves in conventionally neutral wind farms, J. Fluid Mech., 814, 95-130, doi:10.1017/jfm.2017.11, 2017. 
Apsley, D. D. and Castro, I. P.: A limited-length-scale $k-\varepsilon$ model for the neutral and stably-stratified atmospheric boundary layer, Bound.-Lay. Meteorol., 83, 75-98, 1997.

Blackadar, A. K.: The vertical distribution of wind and turbulent exchange in a neutral atmosphere, J. Geophys. Res., 67, 30953102, 1962.

Calaf, M., Meneveau, C., and Meyers, J.: Large eddy simulation study of fully developed wind-turbine array boundary layers, Phys. Fluids, 22, 015110, doi:10.1063/1.3291077, 2010.

Churchfield, M., Wang, Q., Scholbrock, A., Herges, T., Mikkelsen, T., and Sjöholm, M.: Using High-Fidelity Computational Fluid Dynamics to Help Design a Wind Turbine Wake Measurement Experiment, J. Phys. Conf. Ser., 753, 1-13, 2016.

Dörenkämper, M., Witha, B., Steinfeld, G., Heinemann, D., and Kühn, M.: The impact of stable atmospheric boundary layers on wind-turbine wakes within offshore wind farms, J. Wind Eng. Ind. Aerod., 144, 146-153, 2015.

Frandsen, S. T.: Turbulence and turbulence-generated structural loading in wind turbine clusters, Ris $\emptyset-\mathrm{R}-1188(\mathrm{en})$, Ris $\emptyset$ National Laboratory, 2007.

Frandsen, S. T., Jørgensen, H. E., Barthelmie, R., Rathmann, O., Badger, J., Hansen, K., Ott, S., Réthoré, P.-E., Larsen, S. E., and Jensen, L. E.: The Making of a Second-generation Wind Farm Efficiency Model Complex, Wind Energy, 12, 445-458, doi:10.1002/we.351, 2009.

Jonkman, J., Butterfield, S., Musial, W., and Scott, G.: Definition of a 5-MW Reference Wind Turbine for Offshore System Development, Tech. rep., National Renewable Energy Laboratory, 2009.

Koblitz, T., Bechmann, A., Sogachev, A., Sørensen, N., and Réthoré, P.-E.: Computational Fluid Dynamics model of stratified atmospheric boundary-layer flow, Wind Energy, 18, 75-89, 2015.

Lu, H. and Porté-Agel, F.: Large-eddy simulation of a very large wind farm in a stable atmospheric boundary layer, Phys. Fluids, 23, 1-19, doi:10.1063/1.3589857, 2011.

Magnusson, M. and Smedman, A. S.: Influence of Atmosphertic Stability on Wind Turbine Wakes, Wind Engineering, 18, 139152, 1994.

Michelsen, J. A.: Basis3D - a platform for development of multiblock PDE solvers, Tech. Rep. AFM 92-05, Technical University of Denmark, Lyngby, Denmark, 1992.

Mikkelsen, R.: Actuator Disc Methods Applied to Wind Turbines, $\mathrm{PhD}$ thesis, Technical University of Denmark, Mek Dept., Lyngby, Denmark, 2003.
Mitraszewski, K. S.: Study of the wall effect at off shore wind farms, Master's thesis, Technical University of Denmark, 2012.

Orr, A., Hunt, J., Capon, R., Sommeria, J., Cresswell, D., and Owinoh, A.: Coriolis effects on wind jets and cloudiness along coasts, Weather, 60, 291-299, 2005.

Patankar, S. V. and Spalding, D. B.: A calculation procedure for heat, mass and momentum transfer in three-dimensional parabolic flows, Int. J. Heat Mass Tran., 15, 1787-1806, 1972.

Sogachev, A., Kelly, M., and Leclerc, M. Y.: Consistent TwoEquation Closure Modelling for Atmospheric Research: Buoyancy and Vegetation Implementations, Bound.-Lay. Meteorol., 145, 307-327, 2012.

Sørensen, N. N.: General purpose flow solver applied to flow over hills, $\mathrm{PhD}$ thesis, Ris $\emptyset$ National Laboratory, Roskilde, Denmark, 1994.

Sørensen, N. N., Bechmann, A., Johansen, J., Myllerup, L., Botha, P., Vinther, S., and Nielsen, B. S.: Identification of severe wind conditions using a Reynolds Averaged Navier-Stokes solver, J. Phys. Conf. Ser., 75, 1-13, 2007.

van der Laan, M. P. and Sørensen, N. N.: Why the Coriolis force turns a wind farm wake to the right in the Northern Hemisphere, J. Phys. Conf. Ser., 753, 1-7, doi:10.1088/17426596/753/3/032031, 2016.

van der Laan, M. P., Hansen, K. S., Sørensen, N. N., and Réthoré, P.-E.: Predicting wind farm wake interaction with RANS: an investigation of the Coriolis force, J. Phys. Conf. Ser., 524, 1-12, $2015 a$.

van der Laan, M. P., Sørensen, N. N., Réthoré, P.-E., Mann, J., Kelly, M. C., and Troldborg, N.: The $k-\varepsilon-f_{P}$ model applied to double wind turbine wakes using different actuator disk force methods, Wind Energy, 18, 2223-2240, doi:10.1002/we.1816, 2015b.

van der Laan, M. P., Sørensen, N. N., Réthoré, P.-E., Mann, J., Kelly, M. C., Troldborg, N., Schepers, J. G., and Machefaux, E.: An improved $k-\varepsilon$ model applied to a wind turbine wake in atmospheric turbulence, Wind Energy, 18, 889-907, doi:10.1002/we.1736, 2015c.

Volker, P. J. H.: Wake Effects of Large Offshore Wind Farms - a study of the Mesoscale Atmosphere, PhD thesis, DTU Wind Energy, 2014.

Volker, P. J. H., Badger, J., Hahmann, A. N., and Ott, S.: The Explicit Wake Parametrisation V1.0: a wind farm parametrisation in the mesoscale model WRF, Geosci. Model Dev., 8, 3715-3731, doi:10.5194/gmd-8-3715-2015, 2015. 